\title{
Supine PCNL in Flank-Free Oblique Supine Modified Lithotomy (FOSML) Position: Our Point of Technique
}

\section{Adittya K Sharma* and Rahul Yadav}

Rajiv Gandhi Superspeciality Hospital, Tahirpur, New Delhi, India

*Corresponding author: Adittya K Sharma, Rajiv Gandhi Superspeciality Hospital, Tahirpur, New Delhi, India, Tel: 9015058345; Email: dradityaks@gmail.com

\section{Mini Review \\ Volume 3 Issue 2}

Received Date: May 02, 2018

Published Date: May 11, 2018

\section{Abstract}

Objective: There are various positions described for supine PCNL but ideal position for supine PCNL remains debatable. To describe technique of Percutaneous Nephrolithotomy in Flank-free Oblique Supine Modified Lithotomy (FOSML) Position.

Material and Method: Patients with Renal stones $>1 \mathrm{~cm}$ were selected to undergo Percutaneous Nephrolithotomy. After due approval from Research review committee and necessary informed consent, patients were taken up for surgery and given Spinal or General anaesthesia. First standard lithotomy position was given and torso of patient was turned lateral to opposite side. Shoulders remain perpendicular to table and supported by backrest. Ipsilateral Buttock allowed to rest on an inflatable Gel pad and ipsilateral leg extended at the hip to open up the flank. Gel pad under buttock, is inflated to get desired elevation of flank. Final position is Flank free Oblique Supine Modified lithotomy Position.

Result: This position helps in quick positioning after anaesthesia induction. One time painting and draping required for entire surgery and simultaneous lower tract (for cystoscopy and ureteric catheterization) and upper tract (puncture and stone removal) access is possible throughout the surgery. This position gives all the advantages of other supine PCNL positions while overcoming limitations like difficult superior calyceal access or spine superimposition.

Conclusion: This position is easy and uncomplicated with various advantages over previously described positions for supine PCNL. We have found it as most versatile position in terms of stone access (both antegrade \& Retrograde) and access to superior calyx.

Keywords: PCNL; Supine PCNL; PCNL under Spinal anaesthesia; FOSML; Kidney stone

\section{Introduction}

Since its first description by Fernstrom and Johansson [1], prone position has been most traditional way, combining a high success rate and acceptable morbidity. But in modern practice its increasingly evident that onesize-fit-all prone position may not be suitable for many of the patients, particularly the morbidly obese, those with 
respiratory compromise, cases with motor neuron or other neurological disorders ruling out general anaesthesia. Supine or Various combinations of Supine lateral positions have been successfully explored; have shown promising results in terms of feasibility safety and stone free rate.

All these positions have had their share of advantages and disadvantages. In a recent review [2,3], all positions for supine PCNL compared on their Merits and demerits. It showed that Bart's Flank free supine position had maximum advantage of simultaneous antegrade and retrograde access with less restriction to scope movement due to free flank.

In our experience we found that accessing superior calyx was little challenging due to medial position of upper pole calyces. Therefore, we modified flank free position by keeping torso more oblique and allowing more space for puncture and scope manipulation. We are presenting our point of technique in Flank free Oblique Supine Modified Lithotomy (FOSML) Position.

Patient Position: (Figure 1) Patient is initially supine for anaesthesia and important Surface landmarks (Ribs, Iliac crest and Posterior axillary lines) are marked. We prefer spinal anaesthesia is preferred unless ruled out by anaesthetist or patient (preference). A part from usual advantages of regional anaesthesia over general anaesthesia, spinal anaesthesia has quicker induction and does not require reversal before shifting patient after surgery. Therefore it is overall beneficial in terms of saving time in a high volume centres. Patient also remains cooperative to commands and positioning.

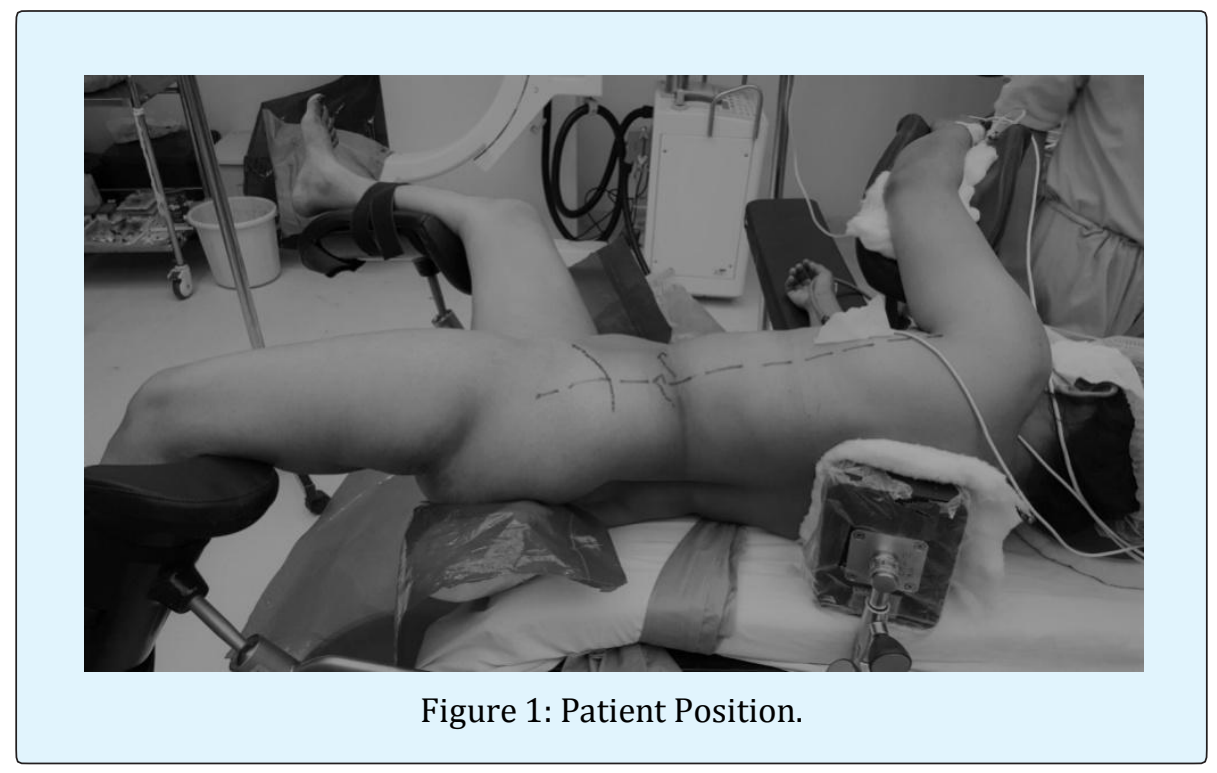

Patient is turned lateral $\left(90^{\circ}\right)$ towards contralateral side and upper back is rested against padded support. Both Lower limbs are supported and fixed on lithotomy poles and ipsilateral buttock rests on Inflatable Gel Pad. Ipsilateral lower limb is slightly extension on hip with knee partially flexed and goes down below the level of table. Contralateral lower limb is in conventional lithotomy position with flexion abduction, external rotation at hip and flexion at knee. Gel Pad under buttock inflated desirably to lift the ipsilateral flank. If simultaneous retrograde access not anticipated then legs can remain straight on table while utilizing flexible Cystoscope for Ureteric catheterization. Over all positioning takes 2-3 mins after anaesthesia.
Puncture: (Figure 2) Using Triangulation Method under Fluoroscopic Guidance, Puncture done (Subcostal or Supracostal depending upon calyceal position) always behind Posterior axillary line. Superior calyceal punctures need to be more medial to adjust for medial position of superior calyces. Oblique position helps in keeping higher punctures medial and allowing more space for scope manipulation. C-Arm is rotated in axial plane to avoid superimposition of calyces with spine. Mixing diluted Betadine or Methylene blue with contrast injected through Ureteric catheter helps in puncture. 


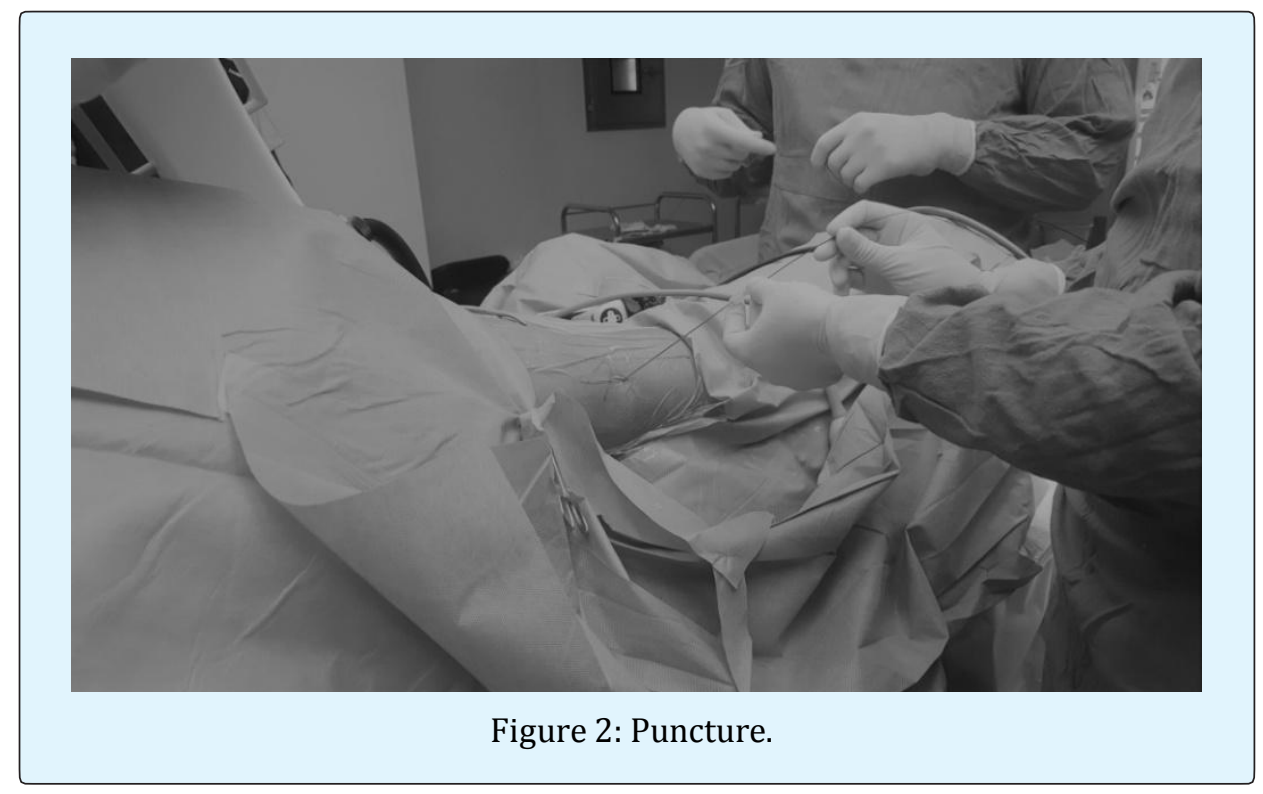

Dilatation: (Figure 3) after Initial puncture skin is incised and single step Alken Needle, cannula passed over guidewire, and additional safety guide wire passed and secured. For standard PCNL we use balloon dilator or single step Amplatz Dilator (24 Fr) over guide-rod.

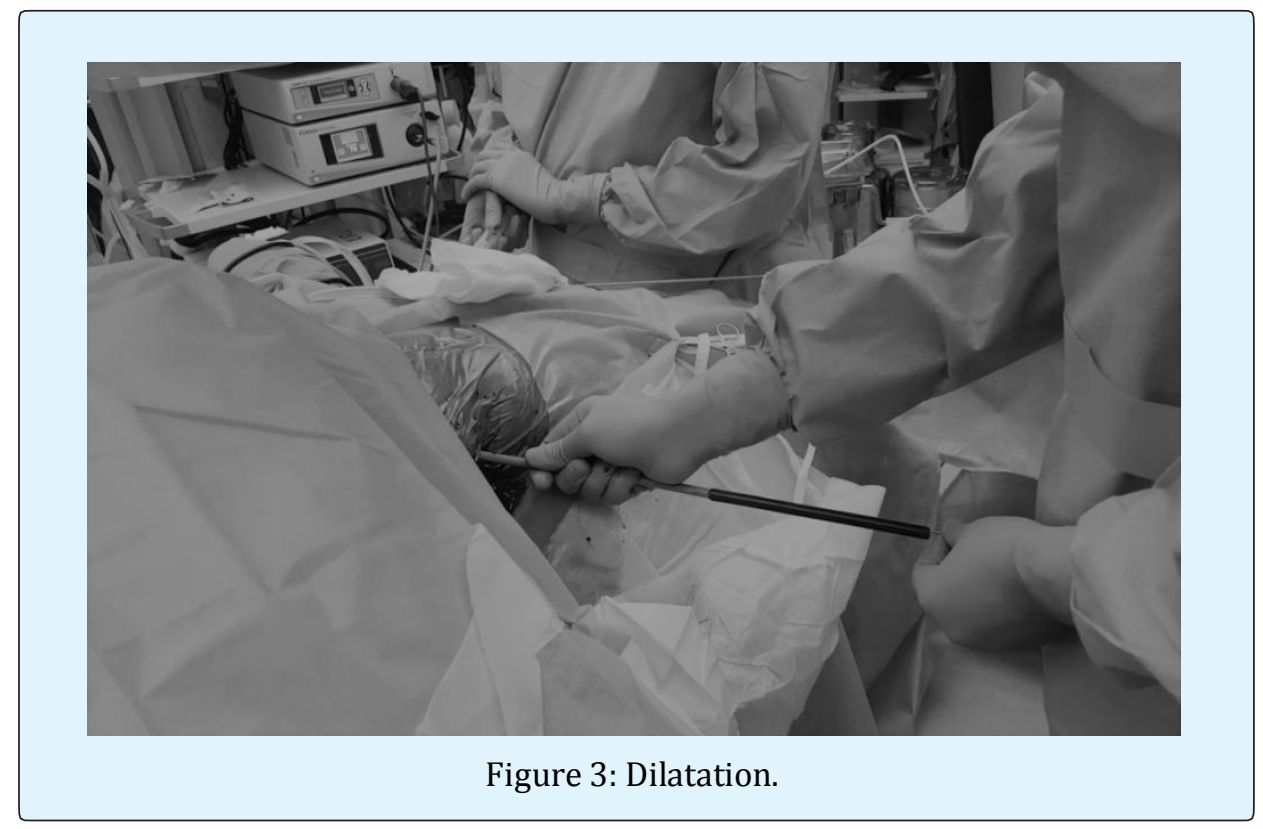

Stone Fragmentation: After passing Amplatz sheath Nephroscope inserted and stone is fragmented using desirable energy source (Pneumatic or Holmium laser). Due to dependant position of other end of nephroscope, the stone fragments pass easily with water outflow with our need of repeated extraction. Due to dependant drainage the pressure within system remains low which reduces chances sepsis. It is always desirable to get a separated puncture if angle between punctured calyx infundibulum and calyx with stone is acute $\left(<90^{\circ}\right)$. For small fragments, which migrate to other calyces, one can use flexible Cystoscope/ Nephroscope for retrieval. Confirmation of clearance done with fluoroscopy in the end. 
Drainage of System: For most uneventful cases we do not keep nephrostomy and we keep ureteric catheter for 24 Hrs. if there was bleeding or residual fragments then we keep Double-J stent along with Nephrostomy tube. Nephrostomy and Foley catheter are removed after $24 \mathrm{hrs}$ followed by discharge. DJS removed after 1-2 weeks.

\section{Discussion}

There are certain advantages of Supine PCNL over Prone position and this FOSML position further increases versatility of this procedure.

\begin{tabular}{|c|c|c|}
\hline & Advantages & Comments \\
\hline 1 & Patient position & Reduced potential risk of musculoskeletal visual complications \\
\hline 2 & Ease of Anaesthesia & Airway access/ Spinal Anaesthesia \\
\hline 3 & Saves OR time & Shorter positioning and recovery time \\
\hline 4 & Special cases. & $\begin{array}{c}\text { Myasthenia grevis/ motor neuron diseases/ Morbid obese/ } \\
\text { respiratory issues }\end{array}$ \\
\hline 5 & Colonic injury & Theoretically less chance compared to prone) \\
\hline 6 & Dependent drainage of system & Spontaneous stone fragment drainage) \\
\hline 7 & Lower system pressure & Reduced potential for post op fever/sepsis \\
\hline 8 & Versatility & Simultaneous ante \& retrograde access / endovision puncture \\
\hline 9 & Relatively lateral puncture & Access to both anterior and posterior calyces \\
\hline 10 & Ease of surgeon & Radiation exposure and ergonomic (sitting position\& relaxed arms) \\
\hline 11 & Type of puncture & $\begin{array}{l}\text { Most punctures are subcostal though Supracostal and superior } \\
\text { calyceal punctures are easily possible with this position. }\end{array}$ \\
\hline 12 & Stone free rate & Equivalent; Can be improved with simultaneous access \\
\hline
\end{tabular}

Table 1: Advantages of Supine PCNL.

There are certain Disadvantages of Supine PCNL compared to prone position.

\begin{tabular}{|c|c|c|}
\hline & Disdvantages & Comments \\
\hline 1 & Learning curve & 8-10 cases; As with any new procedure \\
\hline 2 & Increased mobility of renal unit during dilatation & $\begin{array}{c}\text { As flank is free: (Balloon dilatation or single step } \\
\text { dilatation should be preferred) }\end{array}$ \\
\hline 3 & Apprehension of visceral injury & $\begin{array}{c}\text { Unlikely if retro renal colon is ruled out with Ct } \\
\text { scan and puncture is behind posterior axillary } \\
\text { line }\end{array}$ \\
\hline 4 & Longer access tract & (decreased mobility of nephroscopes) \\
\hline 5 & (Bull's eye not always possible) & Puncture by triangulation only \\
\hline 7 & Superimposition with spine & Can be overcome by rotating C-Arm \\
\hline & $\begin{array}{c}\text { Lesser downward movement of kidney during } \\
\text { inspiration }\end{array}$ & $\begin{array}{c}\text { So puncture manoeuvre by holding inspiration, } \\
\text { less useful compared to prone, specially under } \\
\text { spinal anaesthesia }\end{array}$ \\
\hline
\end{tabular}

Table: 2: Disadvantages of Supine PCNL.

\section{Conclusion}

Supine mPNL is safe with several advantages to patients, surgeon and anesthetist. It gives excellent stone free and saves precious OR time. Our modification to flank free position gives easier access to superior calyx and allows more space for movement of scope. At our center, around $80 \%$ cases of PCNL are done under FOSML position. It has potential of replacing prone approach for most cases of renal stone cases in experienced hands. 


\section{Open Access Journal of Urology \& Nephrology}

\section{References}

1. Fernstrom I, Johansson B (1976) Percutaneous pyelolithotomy. A newextraction technique. Scand J Urol Nephrol 10: 257-259.

2. Karaolides T, Moraitis K, Bach C, Masood J, Buchholz $N$ (2012) Positions for percutaneous nephrolithotomy: Thirty-five years of evolution. Arab J Urol 10(3): 307-316.

3. Zhijian Zhao, Junhong Fan, Yang Liu, Jean de la Rosette, Guohua Zeng (2018) Percutaneous nephrolithotomy: position, position, position!. Urolithiasis 46(1): 79-86. 